\title{
Effects of antigrazing ridges on electron cloud at the BNL Relativistic Heavy Ion Collider warm sections
}

\author{
S. Y. Zhang, ${ }^{*}$ H. C. Hseuh, P. Thieberger, and D. Trbojevic \\ C-A Department, Brookhaven National Laboratory, Upton, New York 11973, USA
}

(Received 21 October 2005; published 14 December 2005)

\begin{abstract}
The antigrazing ridges installed in two Relativistic Heavy Ion Collider (RHIC) warm straight sections have been tested with beam. Significant reduction in electron cloud induced pressure rise has been observed. In addition, the pressure rise pattern at these locations has changed. The effect of antigrazing ridges on the RHIC warm section electron cloud are discussed, as well as possible mechanisms.
\end{abstract}

DOI: 10.1103/PhysRevSTAB.8.123201

PACS numbers: 29.20.Dh, 29.27.Bd

\section{INTRODUCTION}

Electron cloud induced pressure rise in the Relativistic Heavy Ion Collider (RHIC) warm sections is one of beam intensity and luminosity limiting factors [1,2]. Electron cloud effects are observed in RHIC with longer bunch spacings than in other machines. It is suspected that positive ions may enhance multipacting by extending the electron lifetime [3]. Halo scraping may initiate this process by producing positive ions directly or by desorbing gas that is later ionized by the beam or by the electrons. This scenario of beam halo scraping is also consistent with other observations of the electron cloud behavior at RHIC warm sections, i.e., the nonuniform distribution of RHIC electron cloud around the ring, and that the intensity threshold at longer straight sections is lower.

The mechanism is currently under study. One of the investigations is the testing of antigrazing ridges, which were proposed to mitigate the effects of shallow angle incidence of beam, or halo scraping [4]. The original simulations and estimates were performed for gold beams for which the secondary electron and desorption yields are large, but testing with protons is of interest too. The principle is the same. Most of the protons that would otherwise make grazing collisions with the chamber walls collide instead with the ridges, where multiple scattering modify their trajectories.

Just prior to the FY2005 polarized proton run, five ridges were installed in each of two single beam straight sections for beam testing. The shape of these ridges is shown in Fig. 1 and their locations along one of the warm straight sections can be seen in Fig. 2. Pressure rises were monitored by inverted magnetron type cold cathode gauges positioned 10-15 $\mathrm{m}$ apart along the warm beam tubes. They are mounted in well shielded elbows and have sensitivity extending down to $10^{-11}$ Torr with $+/-50 \%$ accuracy.

For this evaluation, beams were injected in the "blue" and "yellow" rings that were similar to beams used in

*Email address: syzhang@BNL.GOV previous beam studies. The electron cloud induced pressure rise at these two locations was significantly reduced. In addition, the pressure rise pattern was also changed. The electron cloud induced pressure rise was highest in the middle of the straight sections without ridges. Now, it became the lowest compared with that at the ends of the sections.

Conventional countermeasures for electron cloud, such as the nonevaporable getter (NEG) coating, bunch gaps, and solenoids, have been studied and applied in RHIC. Antigrazing ridges now provide one more possible means to reduce the pressure rise. For future applications, further studies are needed in terms of ridge sizes, locations, and also of possible machine aperture limitations.

\section{ELECTRON CLOUD AT RHIC WARM SECTIONS}

The electron cloud effects at RHIC warm sections shares many similarities with the ones observed elsewhere [5-7]. For instance, they are affected by bunch intensity, bunch spacing, and bunch gap. The effects of beam scrubbing and solenoids are also observed. Nevertheless, several characteristics of the electron cloud at RHIC warm sections, not similar to others, are also noticed. These are mainly as follows:

(1) The electron cloud occurs at RHIC warm sections with much longer bunch spacings than at other machines with comparable chambers and beam conditions. With $3 \times$ $10^{10}$ protons in a bunch, electron multipacting at some RHIC warm sections happens for bunch spacing of $108 \mathrm{~ns}$. In comparison for electron multipacting to appear with bunch intensity of $3 \times 10^{10}$ to $4 \times 10^{10}$ protons, the bunch spacing is $25 \mathrm{~ns}$ at SPS, and $19 \mathrm{~ns}$ at Tevatron [8]. The lifetime of secondary electrons was measured at PSR as $170 \mathrm{~ns}$ [9], which is consistent with the SPS experience that for bunch spacing of 25,50 , and $75 \mathrm{~ns}$, the intensity threshold is $3 \times 10^{10}, 6 \times 10^{10}$, and $1.2 \times 10^{11}$ protons per bunch, respectively [10]. In Fig. 3, electron multipacting observed at a RHIC interaction region is shown, the bunch spacing is $432 \mathrm{~ns}$, and average bunch intensity is $3.4 \times$ $10^{11}$ protons with the bunch length of $10 \mathrm{~ns}$. 


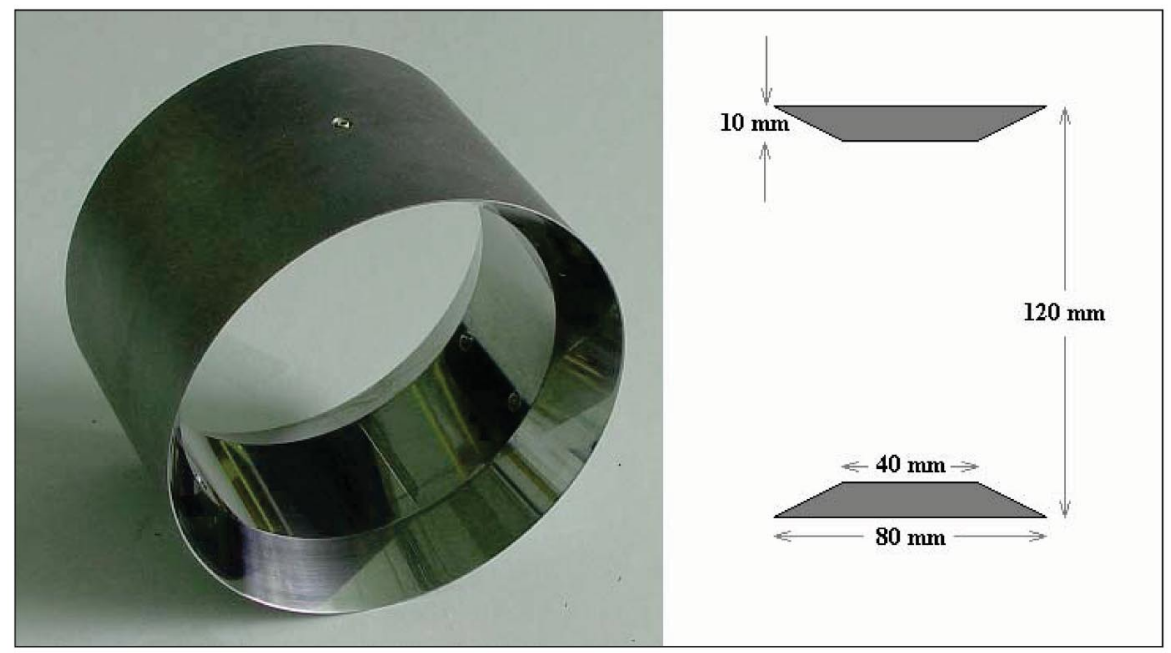

FIG. 1. (Color) Photograph and cross-sectional view of one of the antigrazing rings. At the top of the photograph one of five set screws are visible that are used for positioning and fastening the rings in the beam pipe. The tapering of the ridge edges is introduced to further reduce their already very small impact on ring impedance [4]. A slight taper (not shown) of the inner, 40 mm wide, surface provides a $6 \mathrm{mrad}$ angle with respect to the beam axis to minimize any grazing collisions with this surface.

(2) The RHIC electron cloud at warm sections is nonuniformly distributed in the ring. Figure 4 shows the pressure evolution during injection for all the 12 single (yellow) straight beam sections. Pressure rises are shown at 9 sections ranging from some $10^{-10}$ Torr up to $10^{-6}$ Torr, depending on the locations. There is no pressure rise at all in 3 straight sections.

(3) Electron cloud threshold at longer straight sections is lower than at shorter sections by more than a factor of 2 . In Fig. 5, typical electron cloud induced pressure rises are shown at blue ring, yellow ring, and interaction regions for a beam injection in 2005. Single beam straight sections in blue and yellow rings are $34 \mathrm{~m}$ long, and the interaction region is $17 \mathrm{~m}$ long. It can be seen that the electron cloud threshold at $34 \mathrm{~m}$ long sections of both blue and yellow is about $50 \times 10^{11}$ protons, and for $17 \mathrm{~m}$ long sections it is about $150 \times 10^{11}$ protons.

The longer electron lifetime and nonuniform distribution of RHIC electron cloud at warm sections could be explained by the presence of beam halo scrapping initiated positive ions. The lower threshold at longer straight sections is also consistent with the larger molecular and ionic

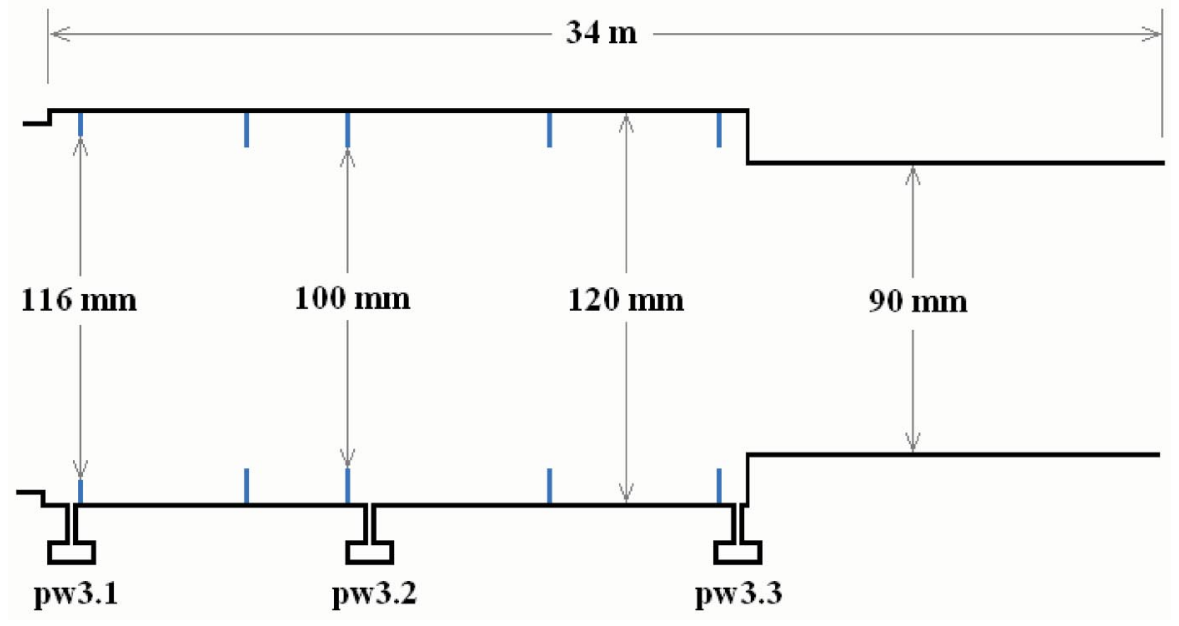

FIG. 2. (Color) Schematic diagram of the Q3-Q4 Yo5 warm section where the antigrazing rings were installed. The longitudinal locations and internal diameters of the stainless steel rings and vacuum pipes are indicated. The horizontal and vertical scales differ by a factor 100. The locations of the rings were determined by practical considerations related to the locations of existing vacuum-tube flanges, and their internal diameters were dictated by concerns against significantly limiting the physical aperture. The labels "pw3" indicate the locations of the vacuum gauges used. Pumping stations are also located at these locations. 

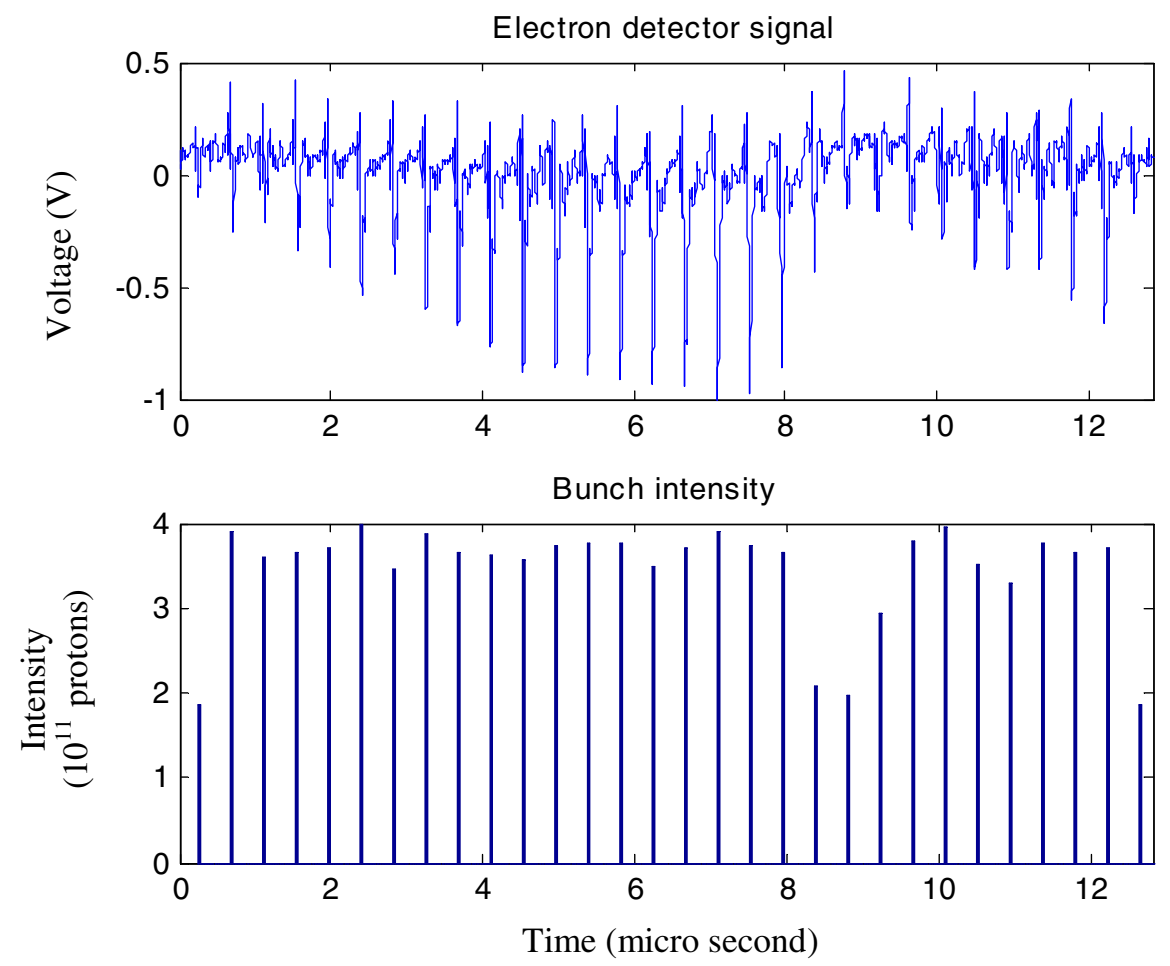

FIG. 3. (Color) Electron multipacting with a bunch spacing of 432 ns at a RHIC interaction region for one revolution period of $12.8 \mu \mathrm{s}$. The $10 \mathrm{~ns}$ long bunches, from blue and yellow rings, meet at the location of the electron detector, with an offset of $3 \mathrm{~ns}$ in time. The total bunch intensity at the electron detector is also shown, and is due to the added contributions of both beams.
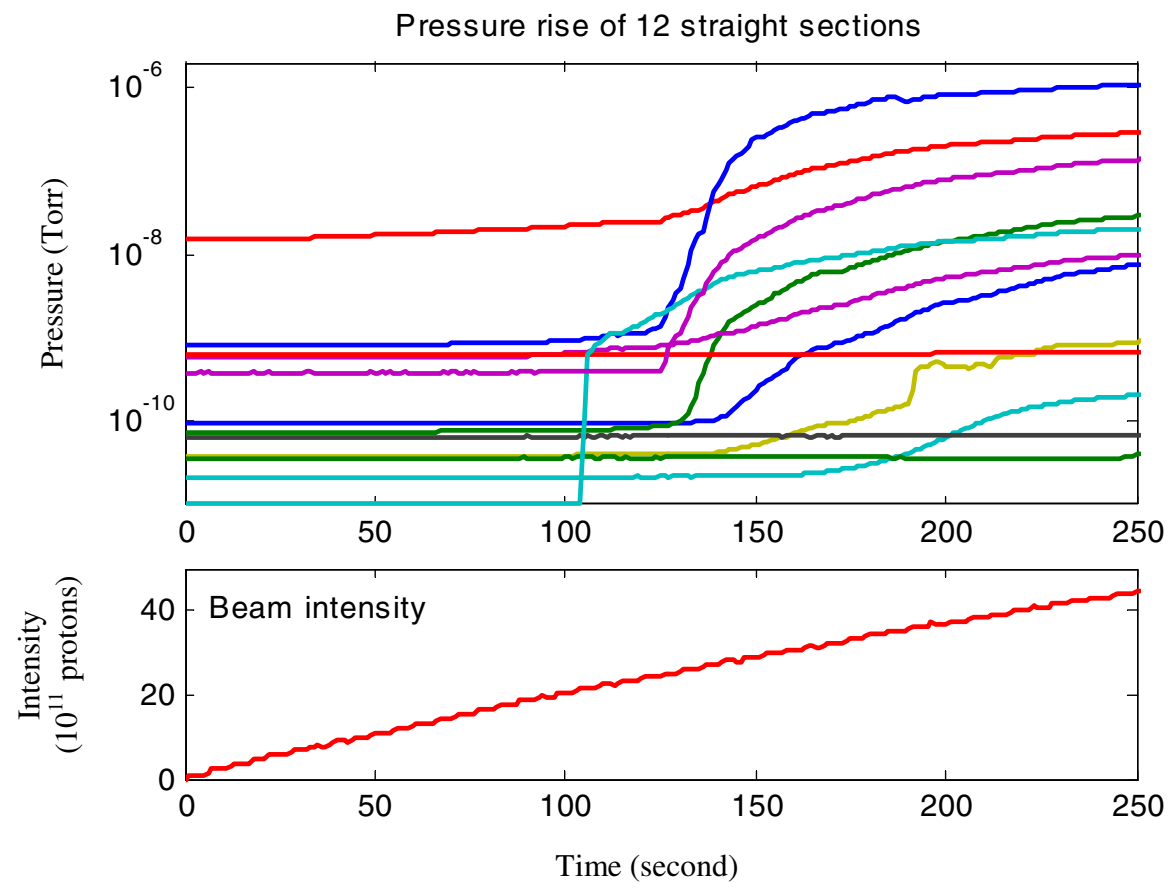

FIG. 4. (Color) Electron cloud induced pressure rise observed during injection in 9 out of the 12 single (yellow) beam straight sections in 2003. About 50 bunches were injected in the 110-bunch mode (bunch spacing of $108 \mathrm{ns),} \mathrm{with} 10^{11}$ protons per bunch. Pressure rise values differ by 4 orders of magnitudes, depending on the locations. Usually in RHIC, one or two sections with the highest pressure rise limit the beam intensity. 

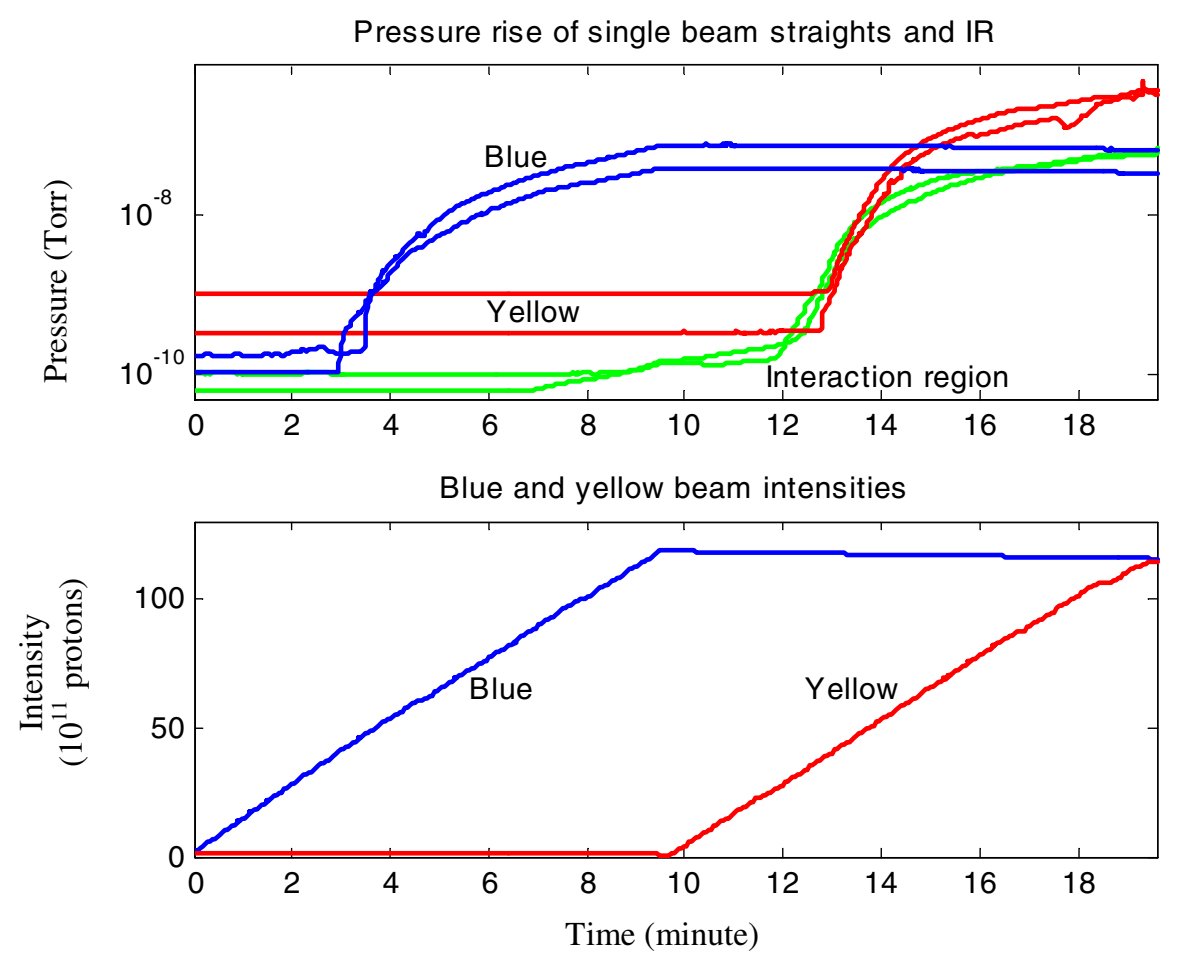

FIG. 5. (Color) Typical electron cloud induced pressure rise in blue and yellow single beam straight sections and interaction regions. The single beam straight sections are $34 \mathrm{~m}$ long, and the interaction region is $17 \mathrm{~m}$ long. Pressure rise in two locations are shown for each type of straight sections. It can be seen that for blue and yellow single beam pipes, electron cloud induced pressure rise starts at $50 \times 10^{11}$ protons, and for interaction regions it starts at about $150 \times 10^{11}$ protons. The beam injection is in the 110 -bunch mode (bunch spacing of $108 \mathrm{~ns}$ ), and the charge per bunch is $1.1 \times 10^{11}$ protons.

yields in shallow angle incidence of the beam at longer pipes.

Direct measurement of these halos and the associated ions is not a straight forward task. As one of the investigations, antigrazing ridges were proposed and installed in RHIC for beam testing. With antigrazing ridges, the shallow angle incidence can be largely prevented [4], and desorption and production of positive ions due to beam halos can be reduced. If the halo scraping initiated positive ions are indeed responsible for multipacting enhancement, lifetime of secondary electrons should be shortened with the ridges installation, and the intensity threshold of electron cloud should be increased.

During the shutdown before the FY2005 polarized proton run, ridges were installed in two single beam straight sections. One is the yellow ring section 5, i.e., Yo5, and the other one is the blue ring section 5, i.e., Bi5 [11].

\section{BEAM TEST RESULTS}

\section{A. Pressure rise reduction}

The key evaluation of the effect of antigrazing ridges is based on the threshold for electron cloud triggering. To compare, similar beams as those used in previous beam studies were injected. Since the study at Bi5 is complicated by the recent installation of NEG coating, the effect of antigrazing ridges there cannot be clearly separated from the contribution of NEG coating. On the other hand, the results at Yo5 have demonstrated that these ridges have effectively reduced the beam induced pressure rise. The pressure rises at Yo5 as function of injected beam intensity, compared with 5 sections without ridges, are shown in Fig. 6.

Yellow beam in 110-bunch mode was injected with a bunch intensity of $1.5 \times 10^{11}$ protons in 2005 , the same as the one in 2004. Among all 12 yellow single beam straight sections, the beam induced pressure rise is much lower in 2005 than in 2004 at 6 sections, where NEG pipes were installed. There are 5 straight sections where the chamber and baking conditions are not changed from 2004 to 2005, these are Yi3, Yi7, Yo9, Yi11, and Yo12. The pressure rises at these locations are comparable in 2005 with that in 2004.

The pressure rise at Yo5 where ridges were installed has been reduced from $5.9 \times 10^{-8}$ Torr to $10^{-9}$ Torr, with a reduction factor of more than 50 . In Table I, the peak pressure rise at Yo5 and at 5 other straight sections without ridges are shown.

A different view of the antigrazing ridges' effect on electron cloud induced pressure rise is shown in Fig. 7, where the pressure rises in 2004, without ridges, and 2005, with ridges, are depicted against the beam intensity. 

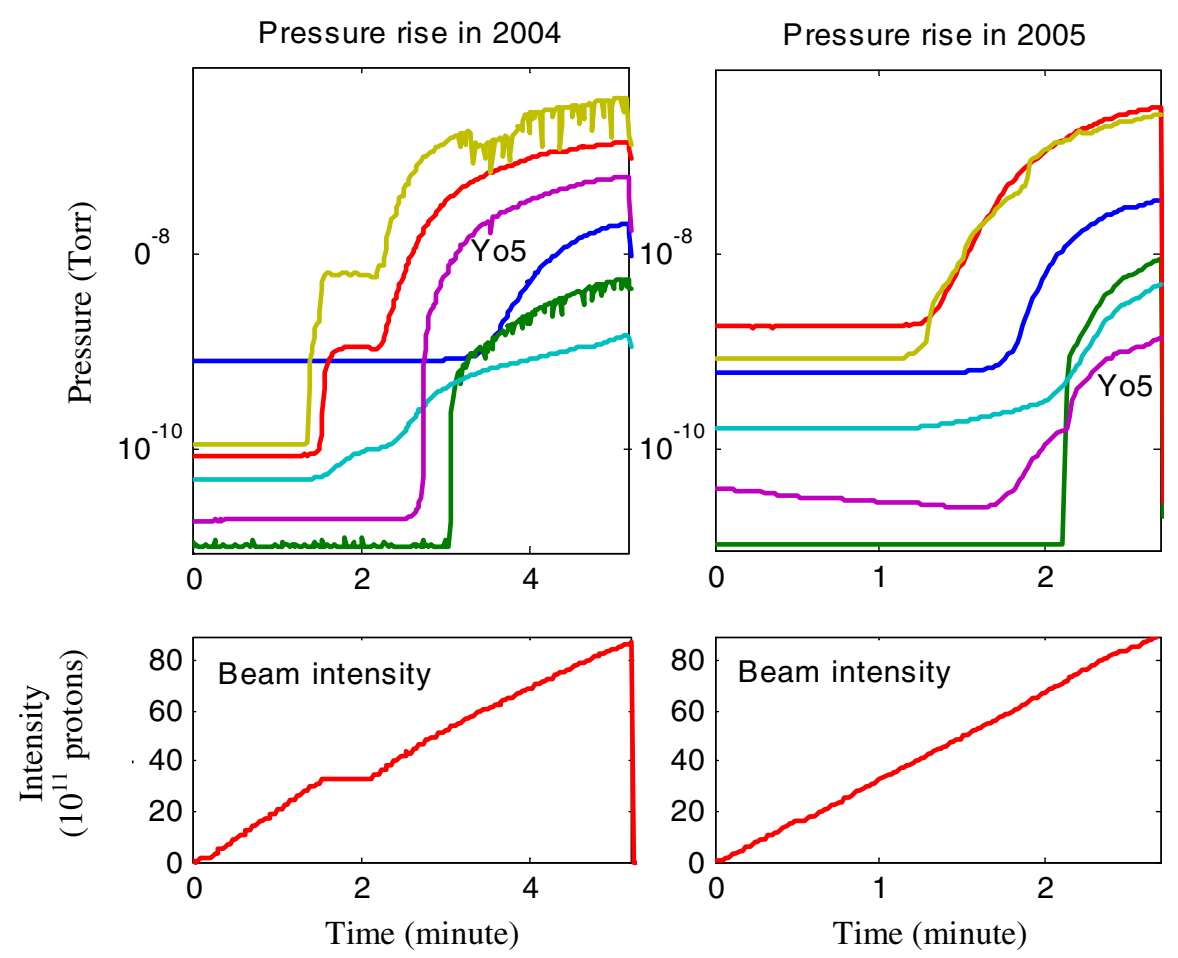

FIG. 6. (Color) The pressure rise at 6 single beam straight sections is shown for yellow beams in the 110-bunch mode and bunch intensity of $1.5 \times 10^{11}$ protons in 2004 and 2005. The purple line is for the Yo5 pressure rise, which is reduced by more than a factor of 50 in 2005 with the ridges, compared with that in 2004 without ridges. The pressure rise at the 5 straight sections without change in chamber conditions, i.e., Yi3, Yi7, Yo9, Yi11, and Yo12, is not much changed from 2004 to 2005.

\section{B. Change of pressure rise pattern}

The electron cloud induced pressure rise is normally the highest at the middle of the single beam straight section, observed at pw3.2, which is the vacuum gauge at the center of straight sections. The pressure rise is about an order of magnitude lower at both pw3.1 and pw3.3, which are at the ends of sections. With similar pumping capacity of ion pumps close to each vacuum gauge, the ones at pw3.1 and pw3.3 are assisted by cryogenic pumping from the cold bore. In addition, the electron multipacting at the middle of straight sections is suspected to be stronger. In Fig. 8, typical pressure rise patterns are shown.

With the ridges, the pressure rise pattern at Yo5 has changed. The pressure rise at the center of the straight section, i.e., pw3.2, is no longer higher than pw3.1 and pw3.3. This pattern is very similar to the ones at the fully or largely NEG pipes equipped straight sections [12]. In Fig. 9, the pressure rise pattern at Yo5 is compared with the one at Yi10, where $31 \mathrm{~m}$ of NEG pipes were installed. The reduction of pressure rise at the center of the section indicates that the electron multipacting was indeed reduced there.

\section{CONCLUSIONS}

The installation of antigrazing ridges to reduce the effects of small-angle collisions produced very significant vacuum performance improvements in the one warm section where no other modifications had been introduced.

TABLE I. Peak pressure rise at Yo5, where ridges were installed for the 2005 run, and at the other 5 straight sections without changes between 2004 and 2005.

\begin{tabular}{lcc}
\hline \hline Location & Peak pressure $\left(10^{-8}\right.$ Torr $) 2004$ & Peak pressure $\left(10^{-8}\right.$ Torr $) 2005$ \\
\hline Yi3 & 0.2 & 0.3 \\
Yo5 & 5.9 & 0.1 \\
Yi7 & 38 & 24 \\
Yo9 & 2 & 3 \\
Yi11 & 0.6 & 0.7 \\
Yo12 & 14 & 29 \\
\hline \hline
\end{tabular}




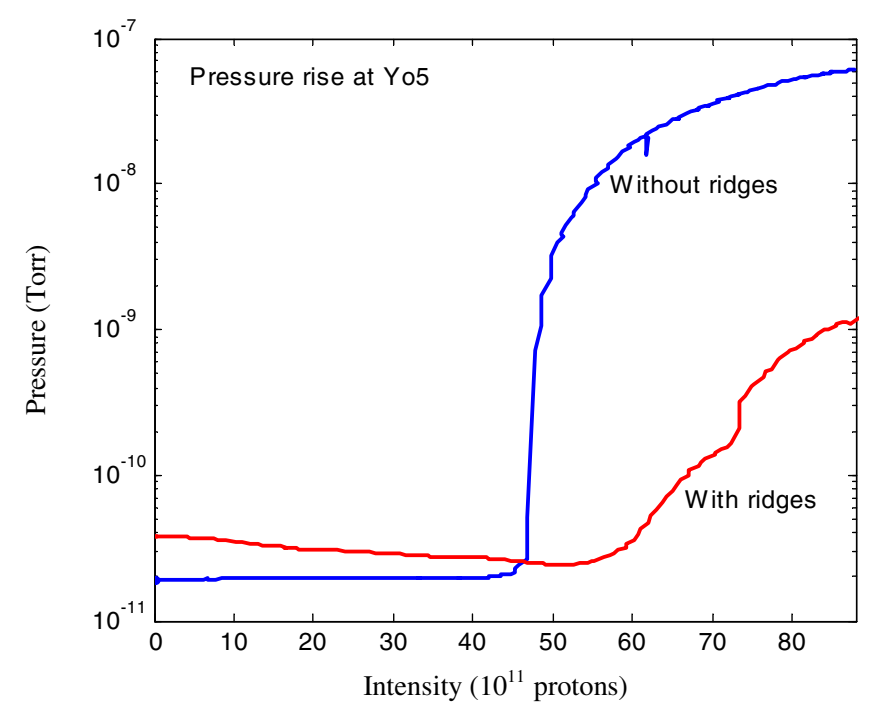

FIG. 7. (Color) The pressure rise at Yo5 is shown against the beam intensity for 2004, without ridges, and 2005, with ridges. Both beam injections are in the 110-bunch mode and bunch intensity of $1.5 \times 10^{11}$ protons. The vacuum and other conditions at Yo5 are not changed from 2004 to 2005, except for the installation of ridges. The early part of the pressure rise of 2005 , with ridges, is a little higher than the one of 2004, which is the remnant of the previous beam injections induced pressure rise.
This improvement was observed with proton beams. Even larger improvements could probably have been observed for gold beams due to their much larger secondary electron and desorption yields.

To overcome the problem with dynamic pressure rise at RHIC warm sections, NEG coating has been proven to be effective [12]. Therefore there are no plans at the moment to install ridges in RHIC, but the test of antigrazing ridges served the following purposes:

The application of NEG pipes at interaction regions is very difficult, mainly due to the problem of activation. Since ridges are effective in raising the electron cloud threshold and in reducing the dynamic pressure rise, there is a potential use of ridges at interaction regions. Also, should NEG saturation or activation requirements become a problem in the future, antigrazing ridges could offer an alternative solution. They may also be of interest for other machines, especially in view of the high cost of NEG coating.

Another motivation for the test of antigrazing ridges is the need of better understanding of the electron cloud at RHIC warm sections. The direct detection of beam halo created positive ions is difficult. The effect of the ridges on reducing the beam induced pressure rise is some evidence that the interaction of beam halo on the wall, and the positive ions generated either directly or through desorbed
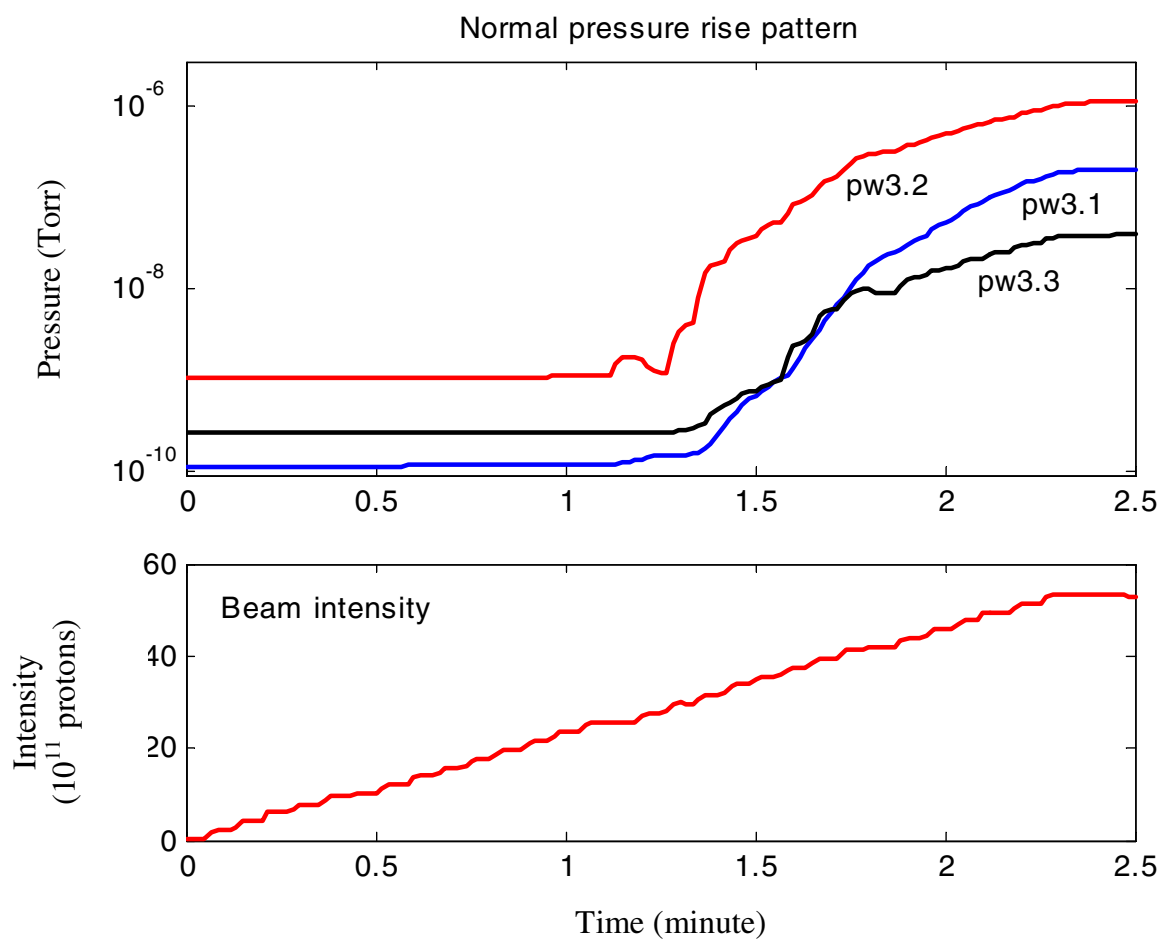

FIG. 8. (Color) Normal pressure rise pattern during injection in single beam straight sections without ridges or NEG coating. The pressure rise is the highest at pw3.2, which is at the middle of the straight section, and about an order of magnitude lower at pw3.1 and pw3.3, which are at the ends of section. With similar pumping capacity of ion pumps close to each vacuum gauge, the ones at pw3.1 and pw3.3 are assisted by cryogenic pumping from the cold bore. In addition, the electron multipacting at the middle of straight sections is suspected to be stronger. 

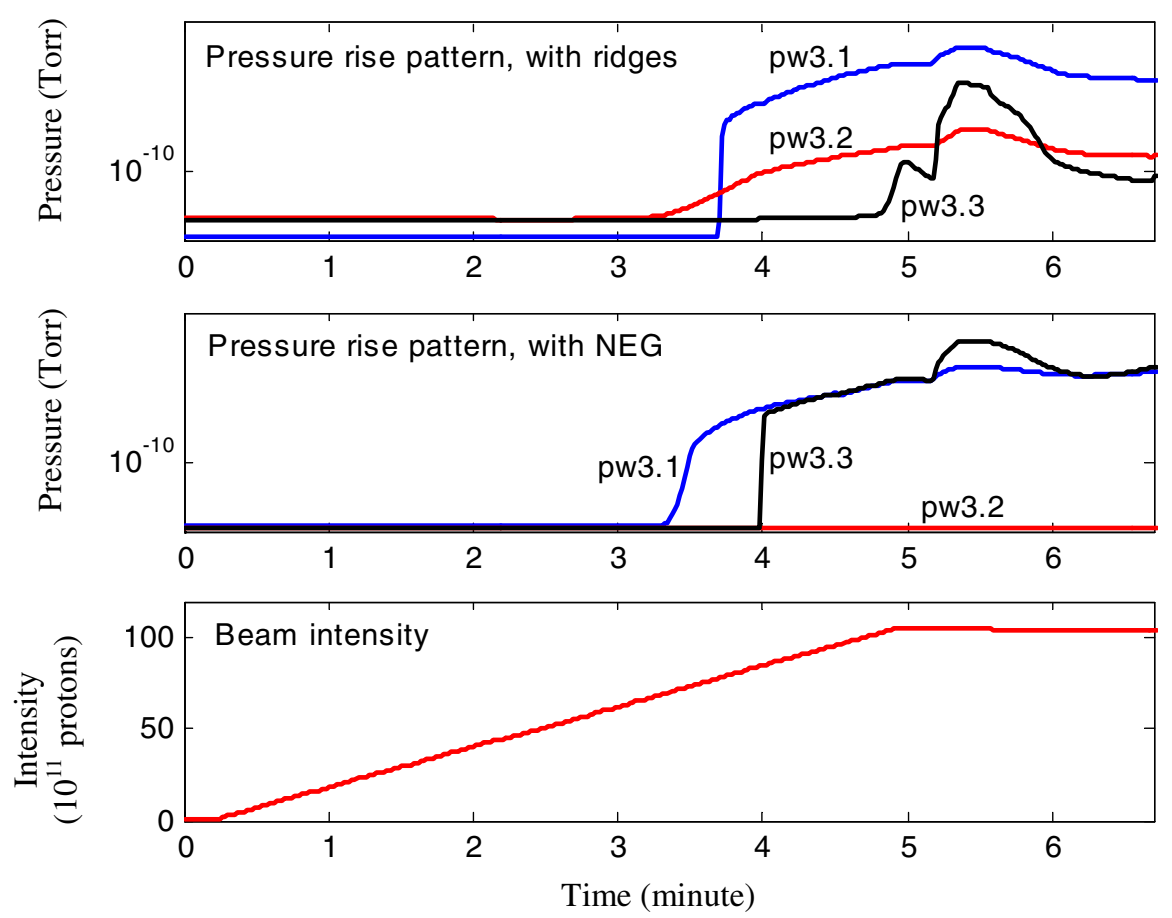

FIG. 9. (Color) Patterns of electron cloud induced pressure rise during injection. Bottom is the yellow beam injection in the 110-bunch mode, with $10^{11}$ protons per bunch. The middle plot shows the electron cloud induced pressure rise at a fully NEG coated straight section. The blue and black lines are for pw3.1 and pw3.3, at the ends of the section, and the red line is for pw3.2, located in the center of the section. Note that the center of the section has the lowest pressure rise. The top plot shows the pressure rise at Yo5 with antigrazing ridges. Note that the pressure rise at the center of this straight section is low, similar to NEG covered sections.

gas ionization, are indeed playing a role in extending the lifetime of secondary electrons, hence making multipacting possible [3] for very long bunch spacings.

\section{ACKNOWLEDGMENTS}

We would like to thank L. Snydstrup for the detailed design of the ridges and for supervision of their fabrication. This work performed under the auspices of the U.S. Department of Energy.

[1] S. Y. Zhang et al., in Proceedings of the Particle Accelerator Conference, Portland, OR, 2003 (IEEE, Piscataway, NJ, 2003).

[2] W. Fischer et al., in Proceedings of ECLOUD'04, Napa, CA, 2004, http://icfa-ecloud04.web.cern.ch/icfa-ecloud04/; Report No. CERN-2005-001, 2005.

[3] P. Thieberger, U. Iriso, and S. Peggs, BNL Report No. C-A/AP/197, 2005; (to be published).
[4] P. Thieberger, W. Fischer, H. C. Hseuh, V. Ptitsyn, L. P. Snydstrup, D. Trbojevic, and S. Y. Zhang, Phys. Rev. ST Accel. Beams, 7, 093201 (2004).

[5] Proceedings of the CERN ECLOUD'02 Workshop, 2002, http://wwwslap.cern.ch/collective/ecloud02/.

[6] Proceedings of the BNL Pressure Rise Workshop, 2003, http://www.c-ad.bnl.gov/icfa/.

[7] Proceedings of ECLOUD'04, Napa, CA, 2004 (Ref. [2]).

[8] F. Zimmermann, in Proceedings of the European Particle Accelerator Conference, Lucerne, Switzerland, 2004 (EPS-AG, Lucerne, 2004).

[9] R. Macek, in Proceedings of the Two Stream Instability Workshop, 2001, http://conference.kek.jp/two-stream/.

[10] G. Arduini et al., in zProceedings of ECLOUD'04, Napa, $C A, 2004$ (Ref. [2]).

[11] S. Y. Zhang et al., in Proceedings of the Particle Accelerator Conference, Knoxville, TN, 2005 (IEEE, Piscataway, NJ, 2005).

[12] S. Y. Zhang, H. C. Hseuh, W. Fischer, H. Huang, and T. Roser, BNL Report No. CAD/AP/220, 2005. 